Cite this: RSC Adv., 2014, 4, 6027

Received 11th October 2013 Accepted 18th December 2013

DOI: 10.1039/c3ra45763j

www.rsc.org/advances

\section{Preparation of nanometre-sized spiral mineral via controlled mineralization using a gel particle as a template $\uparrow$}

\author{
Yuuka Fukui, Saki Nakada and Keiji Fujimoto*
}

We created a local microenvironment for biomineralization by using gel particles. Nanometer-sized and spiral-shaped mineral was produced from the surface of particle aggregates. Also, their pitch and width were controllable by the size of gel particles.

In living systems, mineralization found in nacre and bone is highly regulated within a confined environment provided by organic matrices such as proteins and polysaccharides, resulting in the formation of hierarchical architectures and the emergence of multiple of functions and properties. ${ }^{1,2}$

Of particular note is that organic matters play a crucial role during such mineralization process in both physically and chemically controlled fashions. For example, during mineralization in hard corals, an organic matrix concentrates calcium ions that serve as seeding site for crystal nucleation of calcium carbonate into aragonite polymorph, which protrudes outwards from the organic matrix. ${ }^{3}$ Such organic matrices often exist in the form of hydrogels assembled from proteins, polysaccharides, and glycoproteins to provide the confined space where ions are enriched and inorganic minerals are allowed to grow. It is often said that the local crystallization microenvironment in gel media is distinguished from that in solution by the confinement of solutes to within the pores of the gel network. It is thought that Brownian motion is reduced and thereby a laminar flow and a convective current are suppressed inside the gels, making the diffusion dominant for the mass transport in gel media. This would cause a modification to the crystal growth regimes and numerous studies have been

Center for Chemical Biology, School of Fundamental Science and Technology, Graduate School of Science and Technology, Keio University, 3-14-1 Hiyoshi, Kohoku-ku, Yokohama, 223-8522, Japan. E-mail: fujimoto@applc.keio.ac.jp; Tel: $+81-45-566-1580$

$\dagger$ Electronic supplementary information (ESI) available: Experimental details for preparation of PHEMA gel particles and $\mathrm{CaCO}_{3}$ depositions in the presence of gel particles, transmission electron microscopic images for gel particles, Raman spectra for spiral materials and transmission electron microscopic images for minerals deposited around particle aggregates are included. See DOI: 10.1039/c3ra45763j reported on creation of unique crystal patterns and morphologies both inside and at the surface of gels. ${ }^{4-12}$ Since the gels also serve as sources of chemical functionalities, they act as templates to direct nucleation and induce growth of crystals into desirable sizes and morphologies., ${ }^{5,13,14}$ Herein, we focused on the role of organic polymers in the gel form during mineralization process and introduced the aspect of the colloid chemistry to develop a strategy for designing and synthesizing the minerals by the use of gel particles. Meldrum and Qi et al. have reported on fabrication of calcite crystals within colloidal templates where mineralization was confined to interparticle spaces of polystyrene particles. ${ }^{15-17}$ We previously reported on mineralization using gel particles in which calcium ions were incorporated prior to mineralization. We found that crystal growth of $\mathrm{CaCO}_{3}$ was inhibited inside the gel particles, resulting in formation of amorphous structures. ${ }^{18}$ In this study, we aimed to investigate the environmental effect of such submicron-sized gel particles, which can act as source of ions and also provide the large surface area, on mineralization. It was noteworthy about crystal formation for aggregated gel particles. We observed that low-crystalline minerals with unique spiral structures was grown from the surface of gel particle aggregates and their sizes were in good accord with the size of the particles. Moreover, both the pitch and the width of the spiral material were variable with the size of gel particle. We think that the chemical property of the gel particle surface and the confined space provided by particle aggregates play significant roles in the formation of spiral morphology.

PHEMA gel particle, which is similar to the particle used in our previous study as described above, ${ }^{18}$ was prepared by dispersion polymerization of 2-hydroxyethyl methacrylate (HEMA) and methylenebisacryl-amide (MBAAm) (see ESI $\uparrow$ for details). As a dispersing agent, we used polyvinylpyrrolidone (PVP) or poly(acrylic acid) (PAA), both of which were tethered over the surface of gel particles to render dispersion stability via the steric repulsion or the electrostatic repulsion between ionic groups. The obtained PHEMA gel particles with the diameter of $850 \pm 130 \mathrm{~nm}$ were used for $\mathrm{CaCO}_{3}$ precipitation (Fig. S1 $\dagger$ ). The 
gel particles were alternately immersed in $500 \mu \mathrm{L}$ of $50 \mathrm{mM}$ $\mathrm{Ca}\left(\mathrm{NO}_{3}\right)_{2}$ aqueous solution and $500 \mu \mathrm{L}$ of $100 \mathrm{mM} \mathrm{Na} \mathrm{CO}_{3}$ aqueous solution (solvent displacement method), followed by an incubation at $70^{\circ} \mathrm{C}$ for $24 \mathrm{~h}$.

During this reaction, some of the gel particles seemed to be aggregated to form themselves into clusters, due to dehydration and charge shielding of stabilizing agents, PVP and PAA, caused by the presence of calcium ions. Prior to transmission electron microscopic (TEM) observation, the resultant gel particles and their aggregates were washed with Milli-Q water via the cycles of repeated centrifugation and re-dispersion in order to remove unreacted ions. These were then dried naturally at room temperature and observed by TEM. Fig. 1a shows that in the case of PAA-tethered gel particles, minerals were deposited not only at the surface but inside of gel particles. This is probably because calcium ions bind with carboxylic acid groups of PAA located at the surface and in the inner space of gel particles, so that crystal growth was also allowed inside of gel particles. On the other hand, it was observed that only a few of small and spherical materials were deposited over the surface of individual PVP-tethered gel particles (Fig. 1b). Unlike PAA, PVP is a nonionic polymer with no ability to attract and concentrate ions
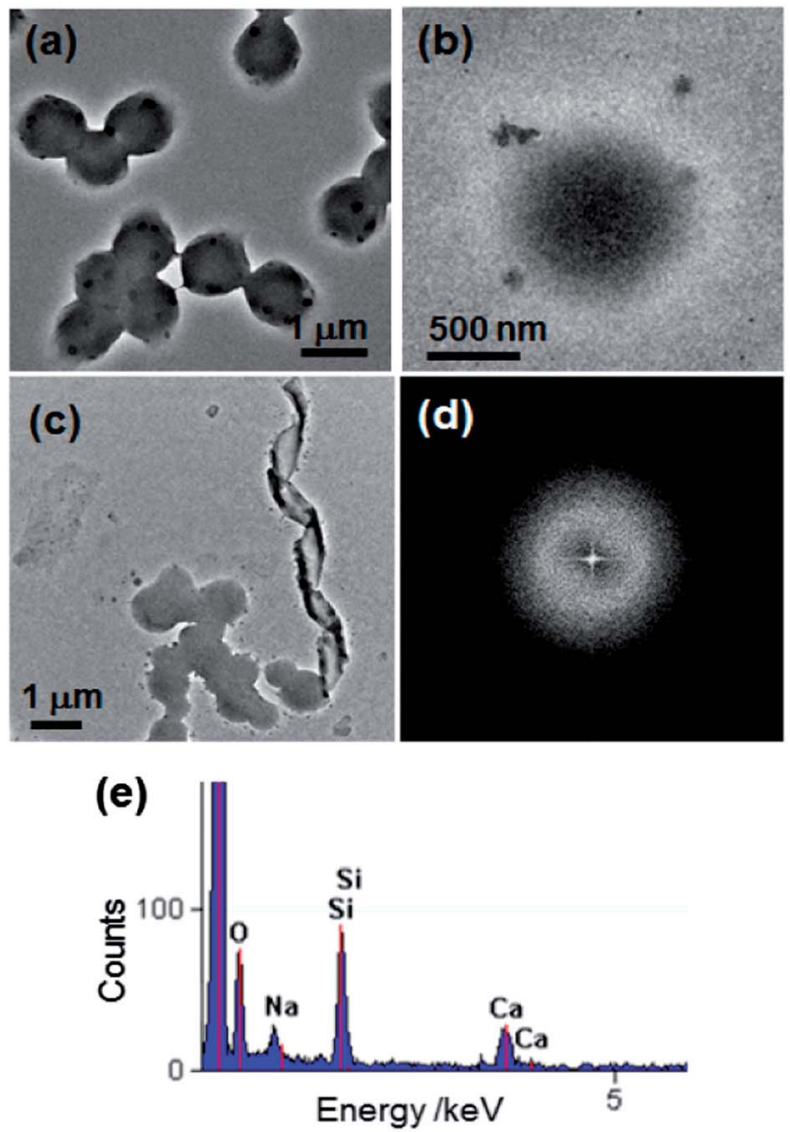

Fig. 1 Transmission electron microscopy images of $\mathrm{CaCO}_{3}$ deposited onto PAA-tethered gel particles (a) and PVP-tethered gel particles (b and $c$ ). Selected area electron diffraction rings (d) and Energy dispersive $\mathrm{X}$-ray analysis spectrum (e) for spiral structure indicate that it is composed of amorphous $\mathrm{CaCO}_{3}$. to promote crystal growth. ${ }^{19}$ Interestingly, it was found that for some of particle aggregates, mineral was precipitated and grown into unique spirals which were protruded outwards from the particle surface as shown in Fig. 1c. Also, the formation of spiral material from almost all the particle aggregates could be observed and one spiral material appeared to be formed from each particle aggregate (data not shown). These spiral structures were analyzed by energy dispersive X-ray spectrometer and peaks for $\mathrm{Ca}$ were detected from all the samples analyzed (Fig. 1e and $\mathrm{S} 2 \dagger$ ). In addition, Raman spectroscopy for spiral structures was performed. A weak and broad band at around $1100 \mathrm{~cm}^{-1}$ which corresponds to symmetric stretching vibration $\left(\nu_{1}\right)$ of the carbonate group was observed (Fig. S3†). From all of these results, we think that spiral structures are composed of $\mathrm{CaCO}_{3}$ with low crystallinity. ${ }^{20}$ Selected area diffraction (SAED) analysis of magnified areas was also conducted to analyze the crystallinity of $\mathrm{CaCO}_{3}$ and Fig. 1d shows that electron diffraction ring and spots were not observed in the electron diffraction pattern of crystals, also suggesting that the spiral-shaped $\mathrm{CaCO}_{3}$ possess low-crystalline structure. It was noteworthy that the size scale for obtained spiral material was similar to that of the gel particles, strongly suggesting that each particle could act as a template for mineralization. In order to clarify this hypothesis, we investigated the effect of the particle size on the formation of spiral crystals. By changing the polymerization periods from $19 \mathrm{~h}$ to $6 \mathrm{~min}$ and $30 \mathrm{~min}$, we could obtain PHEMA gel particles having hydrodynamic diameters of $470 \pm 60$ and $730 \pm 110 \mathrm{~nm}$, respectively. When these particles were utilized as templates for the formation of $\mathrm{CaCO}_{3}$, it could be seen from TEM images that both the width and the pitch of spiral materials changed with respect to the size of particles (Fig. 2). We measured the width
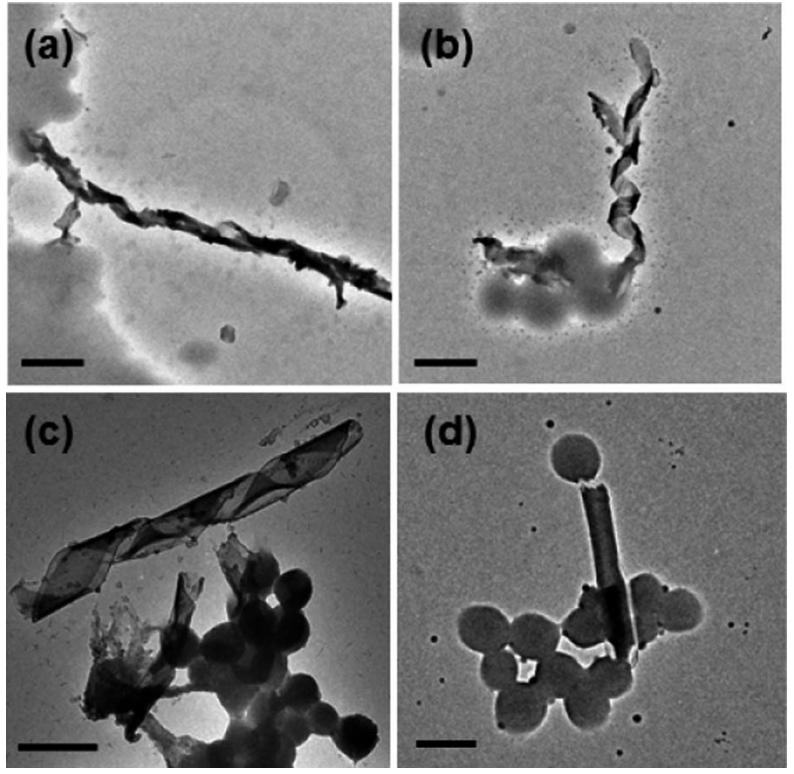

Fig. 2 Transmission electron microscopy images of spiral $\mathrm{CaCO}_{3}$ deposited onto PVP-tethered gel particles with hydrodynamic diameters of $470 \mathrm{~nm}$ (a), $730 \mathrm{~nm}$ (b) and $850 \mathrm{~nm}$ (c and d). Tubular $\mathrm{CaCO}_{3}$ was observed when the reaction system was stirred at 700 rpm during incubation (d). All scale bars are $1 \mu \mathrm{m}$. 
and the pitch of spiral materials in order to clarify the relationship between the particle size and the shape of $\mathrm{CaCO}_{3}$ in a quantitative way. With increasing the particle size, the average width became wider. On the other hand, there was a little tendency between the particle size and the pitch of spiral materials and it is seemed that the shorter the pitch of spirals, the larger the particle size (Table 1). This suggests that the particle surface acts as a template for spiral formation. We think that the curvature of the particle surface would be an important parameter to determine the size and the shape of spiral precipitates. In order to elucidate the possible mechanism for formation of spiral morphology, we performed several experiments as described below. Instead of gel particles, mineralization was carried out in the presence of submicron-sized solid particles, onto which PVP molecules were adsorbed prior to mineralization. Some of the particles were found to be aggregated after the mineralization process and Fig. $\mathrm{S} 4 \uparrow$ shows that only a few of small-sized precipitates were observed at the periphery of particles and within some interparticle spaces. This is because both PVP and solid particles were not able to retain sufficient ions for crystal growth. Therefore, it should be noted that the feature of gels as a source of ions plays a significant role in spiral formation. As stated above, we think that the microenvironment provided by aggregation of gel particles is also a key for spiral formation. For example, a flow of ions within the interparticle space might be suppressed, causing retardation in ion supply to the reaction center. Hence, this would affect the local micro-environment for mineralization such as ion concentrations (supersaturation). To verify the effect of the rate of ion diffusion on mineralization, the reaction system was stirred at $700 \mathrm{rpm}$ during the mineralization process. As a result, we could no longer observe the spiral morphology but instead tubular-shaped materials (Fig. 2d). This is probably due to an increase in the rate of crystal nucleation and growth caused by promotion of ion supply to the reaction center. There have been several studies on mechanisms concerning the formation of helical or spiral $\mathrm{CaCO}_{3} \cdot{ }^{21-30}$ An emergence of inorganic helical structures was often discussed in terms of chemical factors such as presence of chiral macromolecules and physical factors such as diffusion-limited field provided by gel media. Yamamoto et al. showed that macroscopic mirror-imaged helical growth was generated in the presence of the chiral peptides. ${ }^{31}$ Mann et al. reported that adsorption of poly $\alpha, \beta$-aspartic acid at high surface concentrations on the crystal faces caused diffusion gradients in surrounding medium and thereby inducing screw dislocation along the vaterite $c$-axis. ${ }^{23}$ In our report, any chiral molecules was not required for the formation of helical structures. Thus, we think that the diffusion-limited environment provided by gel particle aggregates would be a key for helical formation. It can

Table 1 Relationship between the particle size and width or pitch of spiral $\mathrm{CaCO}_{3}$

Particle size $/ \mathrm{nm}$

Width/nm

Pitch/nm
$470 \pm 60$

$400 \pm 101$

$2480 \pm 1696$
$730 \pm 110$

$473 \pm 155$

$1915 \pm 1004$
$850 \pm 130$

$571 \pm 143$

$1528 \pm 290$ also be interpreted from our results that the presence of particle aggregates composed of PVP-tethered submicron-sized gel particles is crucial for formation of spiral-shaped material. In addition, any formation of spirals could not be observed for individual gel particle and solid particle aggregates. So, we think that both the inner phase of gel particles and the confined space created between particles would act as a reservoir to supply calcium ions to the reaction front at a slow speed. As carbonate ions are initially present in the outer phase of particle aggregates, they would most likely encounter calcium ions at the outermost surface of particle aggregates where nucleation takes place. Because PVP acts as an inhibitor, further growth of mineral should be prevented along the particle surface. Eventually, the reaction and assembly of nanocrystals could proceed apart from the surface, leading to the protrusion and the spiral growth of minerals from the particle aggregates. Formation mechanism of a micrometer-sized helical structure of triclinic crystals in gel media was reported by Imai et al., where they discussed that formation of helical morphology was related to twisted and rotated assembly of tilted crystal subunits, which are favored due to concentration gradients induced by highly diffusion-limited conditions in gel media. ${ }^{30}$ Such helical formation governed by a highly diffusion-limited environment might be applicable to our case. In addition to the inside of the gel, the diffusion of ions is thought to be limited at the surface of the PVP-tethered gel particle and the interparticle space provided by particle aggregates. As described above, spiral material was indicated to be composed of nanocrystalline structures. Therefore, we anticipate that nanocrystals could be accumulated in diffusion-limited environments created by the gel particle aggregates to form the spiral morphology. This report is the first finding on formation of a nanometer-sized helical mineral. We believe that the gel particle used here as a template plays an important role to produce spiral-shaped mineral at the nanometer scale. There still remains a challenging issue to elucidate the precise mechanism.

\section{Conclusions}

In summary, $\mathrm{CaCO}_{3}$ precipitation was carried out in the presence of aggregates composed of several gel particles. It was observed that nanometer-sized and unique spiral-shaped or tubular mineral with low-crystalline structure was produced from the surface of particle aggregates. This is probably due to the diffusion-limited confined space provided by gel aggregates, where mineral was allowed to grow slowly but steadily. Interestingly, the pitch and the width of spiral materials were varied with the size of gel particles. These simple results, although preliminary, would improve our knowledge on the fundamental mechanisms in the biomineralization process as well as its potential importance for developing hybrid nanomaterials and applications.

\section{Notes and references}

1 S. Mann, Biomineralization. Principles and Concepts in Bioinorganic Materials Chemistry, Oxford University Press, Oxford, 2001. 
2 A. Xu, Y. Ma and H. Coelfen, J. Mater. Chem., 2007, 17, 415.

3 T. Mass, J. L. Drake, L. Haramaty, Y. Rosenthal, O. M. E. Schofield, R. M. Sherrell and P. G. Falkowski, PLoS One, 2012, 7, e35049.

4 U. Helbig, J. Cryst. Growth, 2008, 310, 2863.

5 E. Asenath-Smith, H. Li, E. C. Keene, Z. W. Seh and L. A. Estroff, Adv. Funct. Mater., 2012, 22, 2891.

6 Y. Diao, K. E. Whaley, M. E. Helgeson, M. A. Woldeyes, P. S. Doyle, A. S. Myerson, T. A. Hatton and B. L. Trout, J. Am. Chem. Soc., 2012, 134, 673.

7 H. Li and L. A. Estroff, J. Am. Chem. Soc., 2007, 129, 5480.

8 A. Dey, G. de With and N. A. J. M. Sommerdijk, Chem. Soc. Rev., 2010, 39, 397.

9 J. Aizenberg, Adv. Mater., 2004, 16, 1295.

10 T. Sakamoto, A. Oichi, Y. Oaki, T. Nishimura, A. Sugawara and T. Kato, Cryst. Growth Des., 2009, 9, 622.

11 T. Sakamoto, A. Oichi, T. Nishimura, A. Sugawara and T. Kato, Polym. J., 2009, 41, 522.

12 A. Sugawara, T. Ishii and T. Kato, Angew. Chem., Int. Ed., 2003, 42, 5299.

13 S. Weiner, Am. Zool., 1984, 24, 945.

14 S. Weiner and L. Addadi, J. Mater. Chem., 1997, 7, 689.

15 C. Li and L. Qi, Angew. Chem., Int. Ed., 2008, 47, 2388.

16 N. B. J. Hetherington, A. N. Kulak, Y. Kim, E. H. Noel, D. Snoswell, M. Butler and F. C. Meldrum, Adv. Funct. Mater., 2011, 21, 948.

17 W. Yue, A. Kulak and F. C. Meldrum, J. Mater. Chem., 2006, 16, 408.
18 Y. Fukui and K. Fujimoto, J. Mater. Chem., 2012, 22, 3493.

19 W. Hao, S. Qiang, Z. Ying, D. J. Wang and D. F. Xu, J. Cryst. Growth, 2004, 260, 511.

20 U. Wehrmeister, D. E. Jacob, A. L. Soldati, N. Loges, T. Haeger and W. Hofmeister, J. Raman Spectrosc., 2011, 42, 926.

21 Y. Yamamoto, T. Nishimura, T. Saito and T. Kato, Polym. J., 2010, 42, 583.

22 C. A. Orme, A. Noy, A. Wierzbicki, M. T. McBride, M. Grantham, H. H. Teng, P. M. Dove and J. J. DeYoreo, Nature, 2001, 411, 775.

23 S. D. Sims, J. M. Didymus and S. Mann, J. Chem. Soc., Chem. Commun., 1995, 1031.

24 L. A. Gower and D. A. Tirrell, J. Cryst. Growth, 1998, 191, 153. 25 S. H. Yu, H. Colfen, K. Tauer and M. Antonietti, Nat. Mater., 2005, 4, 51.

26 S. Chen, J. Zhu, J. Jiang, G. Cai and S. Yu, Adv. Mater., 2010, 22,540 .

27 J. M. Garcia-Ruiz, E. Melero-Garcia and S. T. Hyde, Science, 2009, 323, 362.

28 J. M. Garcia-Ruiz, S. T. Hyde, A. M. Carnerup, A. G. Christy, M. J. Van Kranendonk and N. J. Welham, Science, 2003, 302, 1194.

29 Y. Oaki and H. Imai, J. Am. Chem. Soc., 2004, 126, 9271.

30 H. Imai and Y. Oaki, Angew. Chem., Int. Ed., 2004, 43, 1363.

31 T. Sugawara, Y. Suwa, K. Ohkawa and H. Yamamoto, Macromol. Rapid Commun., 2003, 24, 847. 